\title{
Quantum Ranging with Gaussian Entanglement
}

\author{
Quntao Zhuang (* \\ Department of Electrical and Computer Engineering and James C. Wyant College of Optical Sciences, \\ University of Arizona, Tucson, Arizona 85721, USA
}

(Received 19 March 2021; accepted 17 May 2021; published 14 June 2021)

\begin{abstract}
It is well known that entanglement can benefit quantum information processing tasks. Quantum illumination, when first proposed, was surprising as the entanglement's benefit survived entanglementbreaking noise. Since then, many efforts have been devoted to study quantum sensing in noisy scenarios. The applicability of such schemes, however, is limited to a binary quantum hypothesis testing scenario. In terms of target detection, such schemes interrogate a single spatiotemporal resolution bin at a time, limiting the impact to radar detection. We resolve this binary-hypothesis limitation by proposing an entanglementassisted quantum ranging protocol. By formulating a ranging task as a multiary hypothesis testing problem, we show that entanglement enables a 6-dB advantage in the error exponent against the optimal classical scheme. Moreover, the proposed ranging protocol can also be used to implement a pulse-position modulated entanglement-assisted communication protocol. Our ranging protocol reveals entanglement's potential in general quantum hypothesis testing tasks and paves the way toward a quantum-ranging radar with a provable quantum advantage.
\end{abstract}

DOI: 10.1103/PhysRevLett.126.240501

Entanglement is one of the most intriguing phenomena promised by quantum physics. As the "spooky action at a distance" unveils itself, it also turns out to be beneficial to various applications. In computation [1,2], entangling multiple qubits in a well-controlled manner enables the efficient computation of difficult problems. In communication [3-5], entanglement enables a higher information transmission rate [6,7] and provides unconditional security [8,9]. In sensing [10-14], entanglement enables the Heisenberg scaling [15] in measuring an identical parameter [16] or even a global property of parameters distributed across different sensors [17-21].

Entanglement is fragile. Noise and loss can easily destroy it, yet surprisingly its operational advantages can survive. For example, the rate of entanglement-assisted (EA) communication can be much larger than the unassisted classical capacity, even for an entanglement-breaking channel that destroys the preshared entanglement, as predicted by the theoretical works [7,22] and recently demonstrated in an experiment [23]. In quantum illumination (QI) [24,25], the target's presence can be probed with a 6 - $\mathrm{dB}$ advantage in the error exponent under the entanglement-breaking noise.

Published by the American Physical Society under the terms of the Creative Commons Attribution 4.0 International license. Further distribution of this work must maintain attribution to the author(s) and the published article's title, journal citation, and DOI.
Many efforts have been devoted to making QI's theoretical advantage practically relevant. Suboptimal receiver designs [26] that enable experimental demonstrations [2729] and a structured optimal receiver design to saturate the quantum advantage [30] have been proposed. To adapt to a radar detection scenario, extensions to the Neyman-Pearson decision strategy [31] and target fading scenarios [32] have been achieved. As the large noise background required by the QI advantage exists only in microwave, demonstration in the microwave domain is also an overall goal [33-35]. However, as pointed out in recent reviews [13,36], a major hurdle that prevents QI being practically advantageous is the fact that it can interrogate the target's presence only in one polarization-azimuth-elevation-range-Doppler resolution bin at a time. It sends signals to a narrow region and judges whether a target moving at a fixed speed is present at a fixed time, while real radar systems in general estimate the polarization properties, azimuth, and elevation angles, range, and velocity (via Doppler effects) of the target. Despite recent theoretical advances in multiary channel discrimination [37,38] that give hope to solving the problem, energetic considerations seem to show that no entanglement advantage can be obtained [39] from that perspective.

In this Letter, we resolve the QI limitation by proposing a quantum ranging protocol enhanced by Gaussian entanglement [40]. First, to go beyond previous studies [37], we develop a precise model for the ranging task, where one sends out a signal pulse and continuously measures at the receiver side to determine the reflection of a target at line of sight. As any ranging task has a finite precision 
requirement, we then formulate ranging as a multiary hypothesis testing problem, where each hypothesis corresponds to the target being in one of the $m \geq 2$ slices of the discretized range. We show that by storing an idler entangled with the signal pulse, the target range can be determined with a 6-dB advantage in the error exponent. Our results on quantum ranging also directly apply to a pulse-position modulated EA classical communication protocol that offers a rate much higher than the classical capacity in the low signal brightness region. We design a practical receiver in the $m=2$ case that enables the entanglement advantage and provides intuition for the optimal receiver design in the general case.

Model of ranging.-We consider the task of determining the distance between an observer and a target along the line of sight. Suppose the observer has a finite precision requirement $\Delta$; then we can divide the line of sight into $m \geq 2$ length- $\Delta$ slices, and model the problem of ranging as a hypothesis testing task between $m$ hypotheses (see Fig. 1). In hypothesis $h$, the target is present in the slice centered at the position $h \Delta$ from the origin.

To determine the range, one can send out a pulse, described by the mode annihilation operator $\hat{a}_{S}$, and wait for the reflected return from the target. The mean photon number of the mode $\left\langle\hat{a}_{S}^{\dagger} \hat{a}_{S}\right\rangle=N_{S}$ is constrained by the source brightness or by the need to avoid revealing the detection attempt. To determine the time of arrival of the returned pulse, one needs to continuously collect light at the receiver side, obtaining the modes $\left\{\hat{a}_{\ell}\right\}_{\ell=1}^{m}$, each arriving at time $t_{\ell}=2 \ell \Delta / c$. In hypothesis $h$, the target is $h \Delta$ away from the observer, and the reflected mode $\hat{a}_{h}$ arrives at the observer after time $t_{h}=2 h \Delta / c$. We can

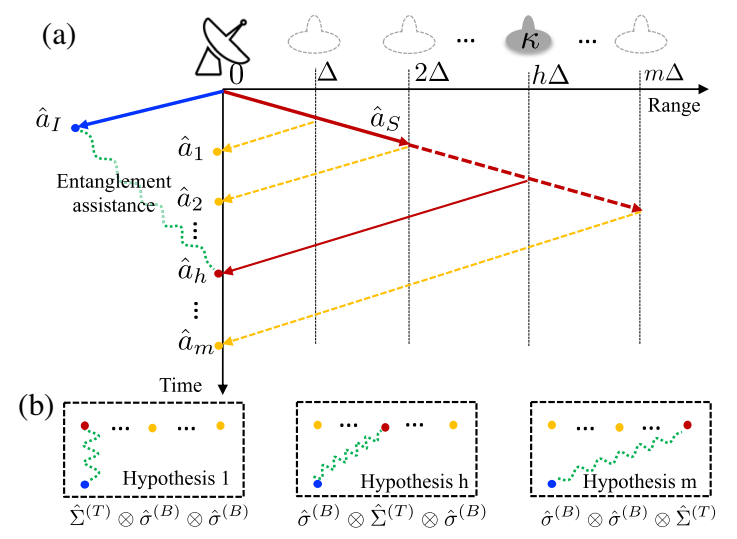

FIG. 1. Schematic of the entanglement-assisted ranging protocol. In (a), the signal mode $\hat{a}_{S}$ (blue) and the idler mode $\hat{a}_{I}$ (red) are initially entangled in a TMSV state. The signal is sent out to probe the range of a target with reflectivity $\kappa$. When the target is at distance $h \Delta$, the mode $\hat{a}_{h}$, highlighted in red, collected at time $t_{h}=2 h \Delta / c$, contains the reflection from the target embedded in noise, while the rest of the collected modes (orange) contain entirely noise. Subplot (b) shows the $m$ possible states in the hypothesis testing problem at the receiver side. In each case, the idler (blue) is correlated with the reflected mode (red). model the reflection by a bosonic thermal-loss channel $\mathcal{L}_{\kappa, N_{B}}$ described by the beam-splitter transform

$$
\hat{a}_{h}=\sqrt{\kappa} \hat{a}_{S}+\sqrt{1-\kappa} \hat{e}_{h},
$$

where $\kappa$ is the target reflectivity and the noise mode $\hat{e}_{h}$ is in a thermal state with $N_{B} /(1-\kappa)$ photons on average. When the returned signal does not arrive at time $t_{\ell}$, the noise mode being collected $\hat{a}_{\ell \neq h}=\hat{e}_{\ell}$ is in a thermal state with mean photon number $N_{B}$.

Now the task of ranging has been reduced to the determination of the returned signal mode $\hat{a}_{h}$ among the entire set of collected modes $\left\{\hat{a}_{\ell}\right\}_{\ell=1}^{m}$. In a classical scheme, the input state of $\hat{a}_{S}$ is assumed to have a positive $\mathrm{P}$ function, as widely considered in the literature $[25,37,41]$. In an entangled scheme, besides sending over the energy-constrained signal mode $\hat{a}_{S}$, one can also keep a locally stored idler $\hat{a}_{I}$ entangled with the signal as depicted in Fig. 1. Similar to the case of QI $[42,43]$, we consider the signal-idler pair in the two-mode squeezed vacuum (TMSV) state (see Ref. [44], Sec. I). Although we expect the TMSV source to be near optimal, proving it is an open problem, e.g., via generalizing approaches in Refs. $[42,43]$. As depicted in Fig. 1(b), the stored idler mode $\hat{a}_{I}$ will still be correlated with the signal mode $\hat{a}_{h}$ returned from the thermal-loss channel $\mathcal{L}_{\kappa, N_{B}}$ in hypothesis $h$, although the initial entanglement might be destroyed. The joint state of $\hat{a}_{I}$ and $\hat{a}_{h}$ has the covariance matrix

$$
\mathbf{V}_{\mathrm{SI}}^{\prime}=\left(\begin{array}{cc}
\left(2 N_{B}+1\right) \mathbf{I}_{2} & 2 \sqrt{\kappa} C_{p} \mathbf{Z}_{2} \\
2 \sqrt{\kappa} C_{p} \mathbf{Z}_{2} & \left(2 N_{S}+1\right) \mathbf{I}_{2}
\end{array}\right),
$$

where $C_{p}=\sqrt{N_{S}\left(N_{S}+1\right)}$ and $\mathbf{I}_{2}, \mathbf{Z}_{2}$ are the Pauli matrices. Here, we have chosen the unit such that the vacuum noise variance is unity. As $\kappa \ll 1$, we have omitted the brightness signature of the target's range in the signal; the results are similar even if we include this difference.

From the potential correlation depicted in Fig. 1(b), it is clear that ranging does not belong to the problem of quantum channel position finding (CPF) defined in Ref. [37]: CPF determines the position of a channel among $m$ channels by inputting $m$ different probes to $m$ channels; while in ranging only a single probe interacts with the target but at an unknown time. For this reason, in EA ranging, it is unclear which signal-idler pair is potentially correlated, while in CPF such pairing between signals and idlers is clear.

Hypothesis testing analyses.-The performance of the above hypothesis testing task is quantified by the error probability. To obtain the best performance, one can optimize the input state under the total photon number constraint $N_{S}$ and the corresponding measurement. One can also use multiple degrees of freedom and send over $M$ modes $\hat{\boldsymbol{a}}_{S} \equiv\left\{\hat{a}_{S}^{(n)}\right\}_{n=1}^{M}$ in each pulse, therefore each portion 
of collected light also contains multiple modes $\hat{\boldsymbol{a}}_{\ell} \equiv$ $\left\{\hat{a}_{\ell}^{(n)}\right\}_{n=1}^{M}$ for each time slice $t_{\ell}$.

In the classical strategy, conditioned on the target range being $h \Delta$, the output state can be written as

$$
\hat{\rho}_{h}^{C}=\left(\otimes_{\ell \neq h} \hat{\sigma}_{\hat{a}_{\ell}}^{(B)}\right) \otimes \hat{\sigma}_{\hat{a}_{h}}^{(T)},
$$

where the background state $\hat{\sigma}^{(B)}$ consists of a product of $M$ thermal states, each with mean photon number $N_{B}$, and the target state $\hat{\sigma}^{(T)}$ is the $M$ mode returned signal embedded in the same thermal background, produced by the thermal-loss channel $\mathcal{L}_{\kappa, N_{B}}$ in Eq. (1). In the entangled scheme, each signal mode $\hat{a}_{S}^{(n)}$ has an idler $\hat{a}_{I}^{(n)}$ stored locally, and the overall return-idler state is

$$
\hat{\rho}_{h}^{E}=\left(\otimes_{\ell \neq h} \hat{\sigma}_{\hat{a}_{\ell}}^{(B)}\right) \otimes \hat{\Sigma}_{\hat{a}_{h} \hat{a}_{I}}^{(T)},
$$

where the correlated output state $\hat{\Sigma}^{(T)}$ has $M$ signal-idler pairs, each in the state described by the covariance matrix $\mathbf{V}_{S I}^{\prime}$ in Eq. (2).

Given the positive operator-valued measure elements $\left\{\hat{\Pi}_{\ell}^{C / E}\right\}_{\ell=1}^{m}$ describing the measurement in the classical (C) or entangled (E) scheme, with each element $\hat{\Pi}_{\ell}^{C / E}$ representing the decision that the target range is $\ell \Delta$, the error probability $P_{C / E}=1-\sum_{\ell=1}^{m} p_{\ell} \operatorname{tr}\left[\hat{\Pi}_{\ell}^{C / E} \hat{\rho}_{\ell}^{C / E}\right]$, where the priors $p_{\ell}=1 / m$ is chosen to be uniform without loss of generality.

Performance of classical schemes.-Using the convexity of the Helstrom limit and the quantum Chernoff bound (QCB) [45-48], we can derive an asymptotically tight expression of the error probability limit of any classical strategy using inputs with a positive $\mathrm{P}$ function (see Ref. [44], Sec. II.A):

$$
\begin{aligned}
P_{C, H} & \sim \frac{m-1}{m} \exp \left[-\frac{2 M \kappa N_{S}}{1+2 N_{B}+2 \sqrt{N_{B}\left(1+N_{B}\right)}}\right] \\
& \simeq \frac{m-1}{m} \exp \left[-\frac{M \kappa N_{S}}{2 N_{B}}\right], \quad \text { when } N_{B} \gg 1,
\end{aligned}
$$

which is tight only in the error exponent. Here the constant $(m-1) / m$ is chosen to match the low signal-to-noise ratio limit with random guess. The limit is achieved by any coherent state input under the proper energy constraint. Furthermore, despite ranging being different from CPF [37] because, as in the classical strategy, no idlers are present, the error probability lower bound of classical CPF (Eq. (10) in Ref. [37]) also applies to classical ranging $P_{C, \mathrm{LB}}=(m-1) / 2 m \times \exp \left[-2 M N_{S} \kappa /\left(1+2 N_{B}\right)\right]$. However this bound is not tight as it gives an error exponent two times larger than that of Eq. (5).

We can compare the asymptotic limit in Eq. (5) with the error-probability performance of the single-mode coherentstate direct detection (DD) strategy $[49,50]$
$P_{C, \mathrm{DD}}=\frac{1}{m} \sum_{k=2}^{m}(-1)^{k} C_{m}^{k} \exp \left[-\frac{\left(1-v^{k-1}\right) \kappa M N_{S}}{\left(1-v^{k}\right) /(1-v)}\right]$,

where $v=N_{B} /\left(N_{B}+1\right)$ and $C_{m}^{k}$ is the number of combinations of $k$ items out of $m$. In the high-noise $N_{B} \gg 1$ and large number of modes $M \gg 1$ limit, $P_{C, \mathrm{DD}} \sim \exp \left(-M \kappa N_{S} / 2 N_{B}\right)$. As it matches the error exponent of Eq. (5), we see that coherent-state DD is the asymptotic optimal classical strategy in terms of the error exponent.

In Fig. 2, we evaluate $P_{C, H}$ (green dashed), $P_{C, \mathrm{LB}}$ (black dashed), and $P_{C, \mathrm{DD}}$ in Eq. (6) (black solid) for various parameters. Indeed, we see that $P_{C, \mathrm{DD}}$ collapses with $P_{C, H}$ for $m=2$ [subplot (a)] and asymptotically agrees with $P_{C, H}$ even for $m>2$ [subplots (b),(c)]. We also numerically evaluate the Helstrom limit in the $m=2$ case and find that $P_{C, H}$ indeed provides the correct scaling. For $m>2$, the numerical evaluation of the Helstrom limit is challenging. We compare it to the performance of the pretty-good measurement (PGM) [37,51-53], which agrees well with the Helstrom limit in the $m=2$ case in Fig. 2(a). For the $m=3$ case, a good agreement between the PGM performance and $P_{C, H}$ can be seen. Therefore, we conclude that $P_{C, H}$ and $P_{C, \mathrm{DD}}$ well characterize the classical performance limit.

Entanglement advantages.-In the EA ranging protocol, one has $M \gg 1$ copies of the identical states in the final idler-return joint state $\hat{\rho}_{h}^{E}$ of Eq. (4). We can therefore apply the QCB for multiple hypotheses $[45,46]$ to obtain the asymptotic error probability. Because of the symmetry of the problem, the error exponent of the multiary hypothesis testing problem is equal to that of discrimination between two three-mode zero-mean Gaussian states with the covariance matrices (see Ref. [44], Sec. II.B):

$$
\begin{aligned}
\mathbf{V}_{12 I}^{(1)} & =\left(\begin{array}{ccc}
\left(2 N_{B}+1\right) \mathbf{I}_{2} & \mathbf{0} & 2 \sqrt{\kappa} C_{p} \mathbf{Z}_{2} \\
\mathbf{0} & \left(2 N_{B}+1\right) \mathbf{I}_{2} & \mathbf{0} \\
2 \sqrt{\kappa} C_{p} \mathbf{Z}_{2} & \mathbf{0} & \left(2 N_{S}+1\right) \mathbf{I}_{2}
\end{array}\right), \\
\mathbf{V}_{12 I}^{(2)} & =\left(\begin{array}{ccc}
\left(2 N_{B}+1\right) \mathbf{I}_{2} & \mathbf{0} & \mathbf{0} \\
\mathbf{0} & \left(2 N_{B}+1\right) \mathbf{I}_{2} & 2 \sqrt{\kappa} C_{p} \mathbf{Z}_{2} \\
\mathbf{0} & 2 \sqrt{\kappa} C_{p} \mathbf{Z}_{2} & \left(2 N_{S}+1\right) \mathbf{I}_{2}
\end{array}\right) .
\end{aligned}
$$

The error exponent can be analytically calculated [48], leading to the asymptotic formula for the Helstrom limit when $N_{B} \gg 1, N_{S} \ll 1$, and $M \gg 1$ as

$$
P_{E, H} \sim \frac{m-1}{m} \exp \left[-\frac{2 M \kappa N_{S}}{N_{B}}\right]
$$




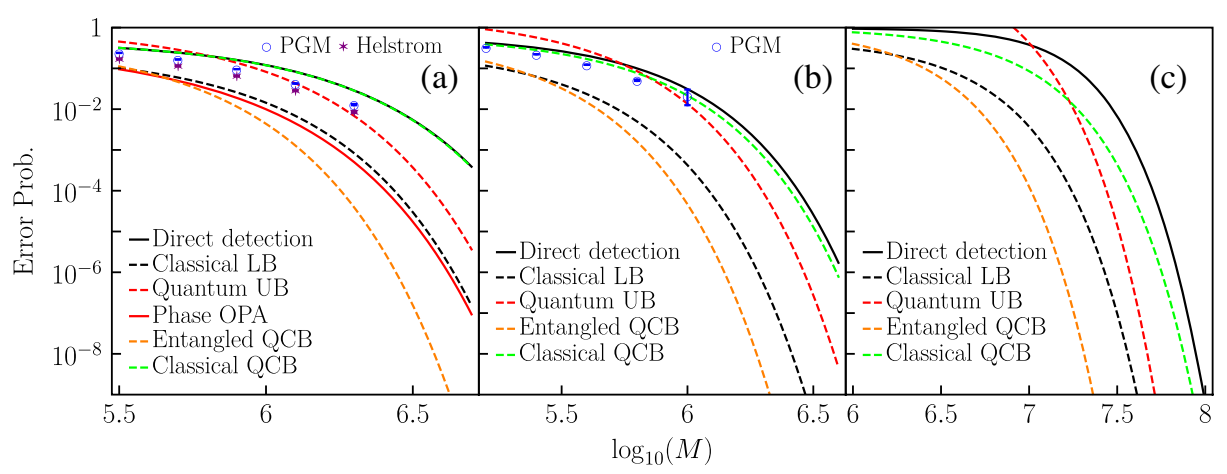

FIG. 2. Error probability performance versus the number of modes $M$ of the quantum ranging protocol compared to the classical schemes. Signal brightness $N_{S}=0.001$ and target reflectivity $\kappa=0.01$. The number of range slices $m$ and the environmental noise $N_{B}$ are chosen as (a) $m=2, N_{B}=3$, (b) $m=3, N_{B}=1$, and (c) $m=50, N_{B}=20$. For the entangled strategy, we evaluate the asymptotically tight quantum Chernoff bound (QCB) $P_{E, H}$ (orange dashed), and an exact upper bound $P_{E, \text { UB }}$ (red dashed). As an upper bound, $P_{E \text {,UB }}$ has values above one in certain region of $M$, where the unity bound of probability dominates. For the classical strategy, we evaluate the QCB $P_{C, H}$ (green dashed), an exact lower bound $P_{C, \mathrm{LB}}$ (black dashed), and the coherent-state direction detection performance $P_{C \text {,DD }}$ (black solid). In (a), we also present the optical-parametric-amplifier-based receiver performance (red solid) in an entangled strategy and the numerical results of the classical Helstrom limit (purple star). In (a),(b), the performance of the pretty good measurement (PGM) for coherent-state inputs is also evaluated numerically compared to the classical QCB. The error bars are from estimation of errors due to the finite photon number cutoff in numerical calculations.

which is tight only in the error exponent. Compared to the optimal classical performance in Eq. (5), we see the EA case in Eq. (8) has a factor of 4 (6-dB) advantage in the error exponent, which is analogous to the entanglement benefit in QI.

We can also derive a PGM-based upper bound [37,51-54] for the Helstrom limit (see Ref. [44], Sec. II.B):

$$
P_{E, H} \leq P_{E, \mathrm{UB}} \simeq(m-1) \exp \left(-\frac{M \kappa N_{S}}{N_{B}}\right)
$$

The error-exponent of $P_{E, \mathrm{UB}}$ is a factor of 2 worse than that of $P_{E, H}$. However, compared to the classical performances in Eqs. (5) and (6), we still see a factor of $2(3-\mathrm{dB})$ advantage in the error exponent.

In Fig. 2, we compare the performances. The entangled upper bound $P_{E \text {,UB }}$ (red dashed) offers rigorous advantages, as well as a scaling advantage in the error exponent, while the asymptotic performance $P_{E, H}$ (orange dashed) provides further advantages (the full expressions are used for evaluation). The QCB results (orange dashed for the entangled case and green dashed for the classical case) are tight in the error exponent, showing a rigorous 6-dB advantage from entanglement. These results confirm the quantum advantage of entanglement in the ranging task, assuming an optimal receiver that jointly measures the entire collected light and the idler.

Entanglement-assisted communication.-It is well known that entanglement preshared between a sender and a receiver enables a higher rate of communication [7]. Yet a protocol that saturates the full advantage is still missing despite recent progress [22,23,55]. In classical signal processing, ranging and pulse-position modulation are closely connected [56]. Indeed, our quantum ranging results can be applied to the design of pulse-position modulated EA communication that provides a much larger advantage. As shown in Fig. 3(a), to send the classical message $h \in[1, m]$, the sender chooses $m$ possible time slices to send the signal part $\hat{a}_{S}$ of the entangled TMSV to the receiver, which collects light continuously to obtain all modes $\left\{\hat{a}_{\ell}\right\}_{\ell=1}^{m}$ corresponding to the $m$ time slices. The receiver then decodes the classical message $\tilde{h}$ by determining which time slice contains the signal from the sender via measuring the collected modes $\left\{\hat{a}_{\ell}\right\}_{\ell=1}^{m}$ jointly with the idler $\hat{a}_{I}$.

In the ranging protocol of Fig. 1, suppose we put all the loss and noise to the receiver side. Then, the target's range
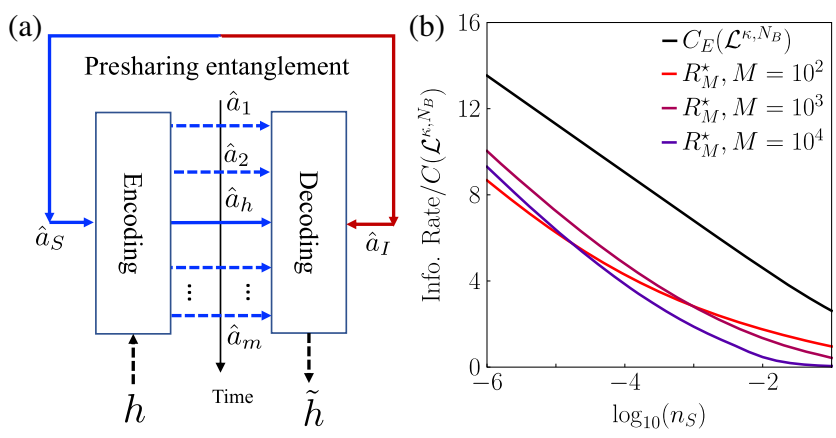

FIG. 3. (a) Schematic of the entanglement-assisted communication protocol. (b) Information rate of the entanglement-assisted communication protocol with $\kappa=0.1$ and $N_{B}=20$. We compare the optimized rates $R_{M}^{\star}$ for the fixed number of repetition modes $M=10^{2}, 10^{3}, 10^{4}$ (red, purple, blue) and the entanglementassisted classical capacity (black) versus the signal average brightness $n_{S}$. 
can be considered as the modulation device of the sender, and the path from the observer to the target as the ideal noiseless channel for entanglement presharing (see Ref. [44], Sec. IV). The same result of Eq. (8) gives the asymptotic optimal decoding error probability, leading to an information rate per mode as $R_{m, M}=I\left(P_{E, H}\right) / M m$, where $I(\cdot)$ is the standard mutual information [see Eq. (41) of Ref. [44]]. We choose the signal total mean photon number $N_{S}=m n_{S}$, giving $n_{S}$ photons being sent per mode per time slice on average. To achieve the best rate, we optimize over the number of time slices $m$ to obtain the optimal rate of EA communication $R_{M}^{\star}=\max _{m} R_{m, M}$.

As benchmarks, we calculate the corresponding classical capacity [57-60] $C\left(\mathcal{L}^{\kappa, N_{B}}\right)$, with the mean photon number constrained to $n_{S}$. As Eq. (8) is asymptotically tight, we consider $M \gg 1$ and plot the ratio of information rate over $C\left(\mathcal{L}^{\kappa, N_{B}}\right)$ in Fig. 3(b) and, indeed, see a great advantage in the low signal brightness region. In fact, when compared to the EA capacity $C_{E}\left(\mathcal{L}^{\kappa, N_{B}}\right)$ (black solid) that upper bounds all possible EA communication rates, we see that the rate $R_{M}^{\star}$ has the optimal scaling $R_{M}^{\star} / C\left(\mathcal{L}^{\kappa, N_{B}}\right) \sim \ln \left(1 / N_{S}\right)$ versus the signal power [22]. Therefore, the receiver design for the ranging protocol would also be able to offer a great advantage in EA communication in the low rate region.

Receiver design.-Here, we provide an opticalparametric amplifier (OPA) [26] based practical receiver design for the ranging problem when $m=2$, with only two groups of collected modes $\left\{\hat{a}_{1}^{(n)}\right\}_{n=1}^{M}$ and $\left\{\hat{a}_{2}^{(n)}\right\}_{n=1}^{M}$ corresponding to the two time slices. One can perform a phase shift on each block of modes and then perform a joint Gaussian operation with the idler modes to obtain $\quad \hat{a}_{I}^{(n) \prime}=\sqrt{G} \hat{a}_{I}^{(n)}+\sqrt{(G-1) / 2} \sum_{\ell=1}^{2} e^{i \ell} \hat{a}_{\ell}^{(n) \dagger}$. To determine the target's range, we measure the total photon number of $\left\{\hat{a}_{I}^{(n) \prime}\right\}_{n=1}^{M}$, with each mode's mean photon number $\left\langle\hat{a}_{I}^{(n) \prime \dagger} \hat{a}_{I}^{(n) \prime}\right\rangle=G N_{S}+(G-1)\left(N_{B}+1\right)+$ $2(-1)^{h} C_{p} \sqrt{G(G-1) \kappa / 2}$ conditioned on hypothesis $h$. Therefore, the hypothesis can be determined from a threshold decision of the photon count. Choosing the optimal gain $G \sim 1+2 \sqrt{N_{S}} / N_{B}$, the error probability performance $P_{E, \text { OPA }} \simeq \exp \left[-M \kappa N_{S} / N_{B}\right] / 2$, when $N_{B} \gg 1, \quad N_{S} \ll 1$ providing a factor of $2(3-\mathrm{dB})$ advantage in the error exponent over the classical limit in Eq. (5), which is confirmed in Fig. 2(a).

Discussions.-We propose a quantum ranging protocol enabled by entanglement to provide a 6- $\mathrm{dB}$ advantage in the error exponent of determining the range among an arbitrary number of possibilities. The receiver design in the general case is an open problem. One potential approach is to design a nondemolition version of the sum-frequencygeneration receiver design [61]. The intuition is that the nondemolition measurement will allow one to use the same idler to interact with all collected modes until the correlated mode is located.
Q.Z. acknowledges the Defense Advanced Research Projects Agency (DARPA) under Young Faculty Award (YFA) Grant No. N660012014029 and Craig M. Berge Dean's Faculty Fellowship of University of Arizona. Q. Z. thanks Saikat Guha, Stefano Pirandola, and Haowei Shi for discussions. Q.Z. acknowledges Jeffrey H. Shapiro for valuable feedback.

*Corresponding author. zhuangquntao@email.arizona.edu

[1] P. Shor, Polynomial-time algorithms for prime factorization and discrete logarithms on a quantum computer, SIAM J. Comput. 26, 1484 (1997).

[2] J. Preskill, Quantum computing in the NISQ era and beyond, Quantum 2, 79 (2018).

[3] N. Gisin, G. Ribordy, W. Tittel, and H. Zbinden, Quantum cryptography, Rev. Mod. Phys. 74, 145 (2002).

[4] F. Xu, X. Ma, Q. Zhang, H.-K. Lo, and J.-W. Pan, Secure quantum key distribution with realistic devices, Rev. Mod. Phys. 92, 025002 (2020).

[5] S. Pirandola, U. L. Andersen, L. Banchi, M. Berta, D. Bunandar, R. Colbeck, D. Englund, T. Gehring, C. Lupo, C. Ottaviani et al., Advances in quantum cryptography, Adv. Opt. Photonics 12, 1012 (2020).

[6] C. H. Bennett and S. J. Wiesner, Communication Via Oneand Two-Particle Operators on Einstein-Podolsky-Rosen States, Phys. Rev. Lett. 69, 2881 (1992).

[7] C. H. Bennett, P. W. Shor, J. A. Smolin, and A. V. Thapliyal, Entanglement-assisted capacity of a quantum channel and the reverse shannon theorem, IEEE Trans. Inf. Theory $\mathbf{4 8}$, 2637 (2002).

[8] C. H. Bennett and G. Brassard, Quantum cryptography: Public key distribution and coin tossing, Theor. Comput. Sci. 560, 7 (2014).

[9] A. K. Ekert, Quantum Cryptography Based on Bell's Theorem, Phys. Rev. Lett. 67, 661 (1991).

[10] V. Giovannetti, S. Lloyd, and L. Maccone, Advances in quantum metrology, Nat. Photonics 5, 222 (2011).

[11] C. L. Degen, F. Reinhard, and P. Cappellaro, Quantum sensing, Rev. Mod. Phys. 89, 035002 (2017).

[12] D. Braun, G. Adesso, F. Benatti, R. Floreanini, U. Marzolino, M. W. Mitchell, and S. Pirandola, Quantum-enhanced measurements without entanglement, Rev. Mod. Phys. 90, 035006 (2018).

[13] S. Pirandola, B. R. Bardhan, T. Gehring, C. Weedbrook, and S. Lloyd, Advances in photonic quantum sensing, Nat. Photonics 12, 724 (2018).

[14] J.S. Sidhu and P. Kok, Geometric perspective on quantum parameter estimation, AVS Quantum Sci. 2, 014701 (2020).

[15] M. Zwierz, C. A. Pérez-Delgado, and P. Kok, General Optimality of the Heisenberg Limit for Quantum Metrology, Phys. Rev. Lett. 105, 180402 (2010).

[16] V. Giovannetti, S. Lloyd, and L. Maccone, Quantum Metrology, Phys. Rev. Lett. 96, 010401 (2006).

[17] W. Ge, K. Jacobs, Z. Eldredge, A. V. Gorshkov, and M. Foss-Feig, Distributed Quantum Metrology with Linear 
Networks and Separable Inputs, Phys. Rev. Lett. 121, 043604 (2018).

[18] T. J. Proctor, P. A. Knott, and J. A. Dunningham, Multiparameter Estimation in Networked Quantum Sensors, Phys. Rev. Lett. 120, 080501 (2018).

[19] Q. Zhuang, Z. Zhang, and J.H. Shapiro, Distributed quantum sensing using continuous-variable multipartite entanglement, Phys. Rev. A 97, 032329 (2018).

[20] Z. Eldredge, M. Foss-Feig, J. A. Gross, S. L. Rolston, and A. V. Gorshkov, Optimal and secure measurement protocols for quantum sensor networks, Phys. Rev. A 97, 042337 (2018).

[21] Z. Zhang and Q. Zhuang, Distributed quantum sensing, arXiv:2010.14744.

[22] H. Shi, Z. Zhang, and Q. Zhuang, Practical Route to Entanglement-Assisted Communication Over Noisy Bosonic Channels, Phys. Rev. Applied 13, 034029 (2020).

[23] S. Hao, H. Shi, W. Li, Q. Zhuang, and Z. Zhang, Entanglement-Assisted Communication Surpassing the Ultimate Classical Capacity, arXiv:2101.07482 [Phys. Rev. Lett. (to be published)].

[24] S. Lloyd, Enhanced sensitivity of photodetection via quantum illumination, Science 321, 1463 (2008).

[25] S.-H. Tan, B. I. Erkmen, V. Giovannetti, S. Guha, S. Lloyd, L. Maccone, S. Pirandola, and J.H. Shapiro, Quantum Illumination with Gaussian States, Phys. Rev. Lett. 101, 253601 (2008).

[26] S. Guha and B. I. Erkmen, Gaussian-state quantum-illumination receivers for target detection, Phys. Rev. A 80, 052310 (2009).

[27] Z. Zhang, M. Tengner, T. Zhong, F. N. C. Wong, and J. H. Shapiro, Entanglement's Benefit Survives an EntanglementBreaking Channel, Phys. Rev. Lett. 111, 010501 (2013).

[28] Z. Zhang, S. Mouradian, F. N. C. Wong, and J. H. Shapiro, Entanglement-Enhanced Sensing in a Lossy and Noisy Environment, Phys. Rev. Lett. 114, 110506 (2015).

[29] E. D. Lopaeva, I. Ruo Berchera, I. P. Degiovanni, S. Olivares, G. Brida, and M. Genovese, Experimental Realization of Quantum Illumination, Phys. Rev. Lett. 110, 153603 (2013).

[30] Q. Zhuang, Z. Zhang, and J. H. Shapiro, Optimum MixedState Discrimination for Noisy Entanglement-Enhanced Sensing, Phys. Rev. Lett. 118, 040801 (2017).

[31] Q. Zhuang, Z. Zhang, and J. H. Shapiro, Entanglementenhanced Neyman-Pearson target detection using quantum illumination, J. Opt. Soc. Am. B 34, 1567 (2017).

[32] Q. Zhuang, Z. Zhang, and J. H. Shapiro, Quantum illumination for enhanced detection of Rayleigh-fading targets, Phys. Rev. A 96, 020302(R) (2017).

[33] S. Barzanjeh, S. Guha, C. Weedbrook, D. Vitali, J.H. Shapiro, and S. Pirandola, Microwave Quantum Illumination, Phys. Rev. Lett. 114, 080503 (2015).

[34] S. Barzanjeh, S. Pirandola, D. Vitali, and J. M. Fink, Microwave quantum illumination using a digital receiver, Sci. Adv. 6, eabb0451 (2020).

[35] C. S. Chang, A. Vadiraj, J. Bourassa, B. Balaji, and C. Wilson, Quantum-enhanced noise radar, Appl. Phys. Lett. 114, 112601 (2019).

[36] J. H. Shapiro, The quantum illumination story, IEEE Trans. Aerospace Electron. Syst. 35, 8 (2020).
[37] Q. Zhuang and S. Pirandola, Entanglement-enhanced testing of multiple quantum hypotheses, Commun. Phys. 3, 103 (2020).

[38] Q. Zhuang and S. Pirandola, Ultimate Limits for Multiple Quantum Channel Discrimination, Phys. Rev. Lett. 125, 080505 (2020).

[39] A. Karsa and S. Pirandola, Energetic considerations in quantum target ranging, arXiv:2011.03637.

[40] C. Weedbrook, S. Pirandola, R. García-Patrón, N. J. Cerf, T. C. Ralph, J. H. Shapiro, and S. Lloyd, Gaussian quantum information, Rev. Mod. Phys. 84, 621 (2012).

[41] S. Pirandola, Quantum Reading of a Classical Digital Memory, Phys. Rev. Lett. 106, 090504 (2011).

[42] R. Nair and M. Gu, Fundamental limits of quantum illumination, Optica 7, 771 (2020).

[43] M. Bradshaw, L. O. Conlon, S. Tserkis, M. Gu, P. K. Lam, and S. M. Assad, Optimal Probes for Continuous Variable Quantum Illumination, arXiv:2010.09156 [Phys. Rev. Lett. (to be published)].

[44] See Supplemental Material at http://link.aps.org/supplemental/ 10.1103/PhysRevLett.126.240501 for details of analyses.

[45] K. Li, Discriminating quantum states: The multiple Chernoff distance, Ann. Stat. 44, 1661 (2016).

[46] M. Nussbaum and A. Szkoła, An asymptotic error bound for testing multiple quantum hypotheses, Ann. Stat. 39, 3211 (2011).

[47] K. M. R. Audenaert, J. Calsamiglia, R. Munoz-Tapia, E. Bagan, L. Masanes, A. Acin, and F. Verstraete, Discriminating States: The Quantum Chernoff Bound, Phys. Rev. Lett. 98, 160501 (2007).

[48] S. Pirandola and S. Lloyd, Computable bounds for the discrimination of Gaussian states, Phys. Rev. A 78, 012331 (2008).

[49] C. Helstrom, Quantum Detection and Estimation Theory, Mathematics in Science and Engineering: A series of monographs and textbooks (Academic Press, New York, 1976).

[50] G. Cariolaro and G. Pierobon, Theory of quantum pulse position modulation and related numerical problems, IEEE Trans. Commun. 58, 1213 (2010).

[51] A. S. Holevo, On asymptotically optimal hypothesis testing in quantum statistics, Theory Probab. Appl. 23, 411 (1978).

[52] P. Hausladen and W. K. Wootters, A 'pretty good' measurement for distinguishing quantum states, J. Mod. Opt. 41, 2385 (1994).

[53] P. Hausladen, R. Jozsa, B. Schumacher, M. Westmoreland, and W. K. Wootters, Classical information capacity of a quantum channel, Phys. Rev. A 54, 1869 (1996).

[54] L. Banchi, S. L. Braunstein, and S. Pirandola, Quantum Fidelity for Arbitrary Gaussian States, Phys. Rev. Lett. 115, 260501 (2015).

[55] S. Guha, Q. Zhuang, and B. A. Bash, Infinite-fold enhancement in communications capacity using pre-shared entanglement, in 2020 ISIT (IEEE, 2020), pp. 1835-1839.

[56] H. L. Van Trees, Detection, estimation, and modulation theory, part I: Detection, estimation, and linear modulation theory (John Wiley \& Sons, New York, 2004).

[57] P. Hausladen, R. Jozsa, B. Schumacher, M. Westmoreland, and W. K. Wootters, Classical information capacity of a quantum channel, Phys. Rev. A 54, 1869 (1996). 
[58] B. Schumacher and M.D. Westmoreland, Sending classical information via noisy quantum channels, Phys. Rev. A 56, 131 (1997).

[59] A.S. Holevo, The capacity of the quantum channel with general signal states, IEEE Trans. Inf. Theory 44, 269 (1998).
[60] V. Giovannetti, R. Garcia-Patron, N. J. Cerf, and A. S. Holevo, Ultimate classical communication rates of quantum optical channels, Nat. Photonics 8, 796 (2014).

[61] Q. Zhuang, Z. Zhang, and J. H. Shapiro, Entanglementenhanced lidars for simultaneous range and velocity measurements, Phys. Rev. A 96, 040304(R) (2017). 